\title{
More on geometry of Krein space $C$-numerical range
}

\author{
Alexander Guterman \\ Faculty of Algebra, Department of Mathematics and Mechanics, Moscow State University, \\ GSP-1, 119991 Moscow, Russia \\ Rute Lemos \\ CIDMA - Center for Research and Development in Mathematics and Applications, \\ Department of Mathematics, University of Aveiro, 3810-193 Aveiro, Portugal \\ Graça Soares \\ Mathematical Center CMAT, Pole CMAT-UTAD, University of Trás-os-Montes and Alto \\ Douro, UTAD, School of Science and Technology, Quinta dos Prados, 5000-801 Vila Real, \\ Portugal, www.utad.pt
}

\begin{abstract}
For $n \times n$ complex matrices $A, C$ and $H$, where $H$ is non-singular Hermitian, the Krein space $C$-numerical range of $A$ induced by $H$ is the subset of the complex plane given by $\left\{\operatorname{Tr}\left(C U^{[*]} A U\right): U^{-1}=U^{[*]}\right\}$ with $U^{[*]}=H^{-1} U^{*} H$ the $H$-adjoint matrix of $U$. We revisit several results on the geometry of Krein space $C$-numerical range of $A$ and in particular we obtain a criteria for the Krein space $C$-numerical range to be a subset of the real line.
\end{abstract}

Keywords: Krein space $C$-numerical range, indefininte inner product, $J$-Hermitian matrix 2010 Mathematics Subject Classification. 15A15, 15A60, 15A86

\section{Introduction}

Let $M_{n}$ denote the algebra of $n \times n$ complex matrices, $I_{n}$ denote the identity matrix of order $n$ and $H \in M_{n}$ be a non-singular Hermitian matrix. Consider $\mathbb{C}^{n}$ endowed with the inner product (possibly indefinite) induced by $H$, that is,

$$
\langle x, y\rangle_{H}=y^{*} H x, \quad x, y \in \mathbb{C}^{n} .
$$

Let $A \in M_{n}$. The Krein space numerical range of $A$ induced by $H$ is defined by

$$
W_{H}(A)=\left\{\frac{\langle A x, x\rangle_{H}}{\langle x, x\rangle_{H}}: x \in \mathbb{C}^{n},\langle x, x\rangle_{H} \neq 0\right\}
$$

Email addresses: guterman@list.ru (Alexander Guterman), rute@ua.pt (Rute Lemos), gsoares@utad.pt (Graça Soares)

Preprint submitted to Applied Mathematics and Computation

November 19, 2018 
If $H=I_{n}$, this set reduces to the classical numerical range of $A$,

$$
W(A)=\left\{x^{*} A x: x \in \mathbb{C}^{n}, x^{*} x=1\right\},
$$

a well-known concept that has been extensively investigated and generalized, playing an important role in the study of matrices and operators. Numerical range is very useful both for pure mathematical problems and for various applied topics, see for example [8], where applications to quantum information science are presented. The set $W(A)$ is compact and convex for any $A \in M_{n}$, see $[6,14]$. In contrast with the classical case, when $H$ is an indefinite matrix, the set $W_{H}(A)$ may be neither closed nor convex, and it is either unbounded or a singleton [10]. There are many results which connect analytic and algebraic properties of a matrix with the geometric properties of its numerical range. There is also a substantial interest in investigating these relations in the Krein space setting. For instance, it is true that:

(C1) $W_{H}(A)$ is a singleton if and only if $A$ is a scalar matrix (cf. [10, Theorem $2.3(\mathrm{~b})])$.

Considering $A^{[*]}=H^{-1} A^{*} H$ the adjoint of $A$ with respect to the inner product induced by $H$,

(C2) $W_{H}(A) \subset \mathbb{R}$ if and only if $A$ is $H$-Hermitian, i.e., $A=A^{[*]}$ (cf. [10, Corollary $2.5(\mathrm{a})]$ ).

In addition to $W_{H}(A)$, we may also consider the sets

$$
\begin{gathered}
W_{H}^{+}(A)=\left\{\langle A x, x\rangle_{H}: x \in \mathbb{C}^{n},\langle x, x\rangle_{H}=1\right\}, \\
W_{H}^{-}(A)=\left\{\langle A x, x\rangle_{H}: x \in \mathbb{C}^{n},\langle x, x\rangle_{H}=-1\right\},
\end{gathered}
$$

which are both convex. The relation $W_{-H}^{+}(A)=-W_{H}^{-}(A)$ clearly holds. If $H$ admits at least one positive eigenvalue, then the results $(\mathrm{C} 1)$ and $(\mathrm{C} 2)$ stated above hold also for the set $W_{H}^{+}(A)$ (cf. [10, Theorem 2.3 (b) and Corollary 2.5 (a), respectively].

Further, we have

$$
W_{H}(A)=W_{H}^{+}(A) \cup W_{-H}^{+}(A) .
$$

Definition 1.1. A subset $S$ of the complex plane is called pseudo-convex if for any pair of points $z, w \in S$, the set $S$ contains either the closed line segment joining $z$ and $w$ or the line defined by $z$ and $w$ except the open line segment joining them.

The set $W_{H}(A)$ is pseudo-convex [10]. If $H$ is positive definite, then by [10, Theorem 2.3(c)] we have

$$
W_{H}(A)=W_{H}^{+}(A)=W\left(H^{1 / 2} A H^{-1 / 2}\right) \quad \text { and } \quad W_{H}^{-}(A)=\emptyset .
$$


There are several generalizations of the classical numerical range, motivated by a number of theoretical and applied problems, such as the $C$-numerical range of $A$, denoted and defined by

$$
W^{C}(A)=\left\{\operatorname{Tr}\left(C U^{*} A U\right): U \in M_{n}, U^{-1}=U^{*}\right\}
$$

for any $A, C \in M_{n}$, which reduces to $W(A)$ when $C$ is a rank one Hermitian orthogonal projection. The previous concept motivates the Krein space $C$-numerical range of $A$ induced by $H$, denoted and defined by

$$
W_{H}^{C}(A)=\left\{\operatorname{Tr}\left(C U^{[*]} A U\right): U \in M_{n}, U^{-1}=U^{[*]}\right\} .
$$

We remark that the invertible matrices $U \in M_{n}$ such that $U^{-1}=U^{[*]}$ are called $H$-unitary and form a group which we will denote by $\mathcal{U}_{H}$. In parallel, the set $V_{H, C}(A)$, consisting of all the elements $\operatorname{Tr}\left(C U^{*} A U\right)$ with $U \in M_{n}$ such that $U^{*} H U=H$, was firstly introduced in [1] and it was then called the $(H, C)$ tracial range of $A$. We remark that $W_{H}^{C}(A)=V_{H, C H^{-1}}(H A)$.

The following elementary properties of the Krein space $C$-numerical range (cf. [1]) are easily verified:

P1. $W_{H}^{C}(A)=W_{H}^{A}(C)$;

P2. $W_{H}^{\left(R^{[*]} C R\right)}\left(U^{[*]} A U\right)=W_{H}^{C}(A)$, for any $H$-unitary matrices $U, R \in M_{n}$;

P3. $W_{H}^{C}\left(\alpha I_{n}+\beta A\right)=\alpha \operatorname{Tr}(C)+\beta W_{H}^{C}(A)$, for any $\alpha, \beta \in \mathbb{C}$;

P4. $W_{H}^{C^{[*]}}\left(A^{[*]}\right)=\left\{\bar{z}: z \in W_{H}^{C}(A)\right\}$.

If the non-singular Hermitian matrix $H$ has signature $(r, n-r)$, that is, $r$ positive and $n-r$ negative eigenvalues, then $J=I_{r} \oplus-I_{n-r}$ is the inertia matrix of $H$. Using Sylvester's law of inertia [7, Theorem 4.5.8], we can easily check that

$$
W_{H}(A)=W_{J}\left(S^{-1} A S\right) \quad \text { and } \quad W_{H}^{C}(A)=W_{J}^{S^{-1} C S}\left(S^{-1} A S\right),
$$

where $S$ is a non-singular matrix, such that $S^{*} H S=J$. Hence, we may focus our attention on Krein space numerical ranges induced by

$$
J=I_{r} \oplus-I_{n-r}, \quad 0 \leq r \leq n .
$$

The $J$-unitary matrices form a locally compact, connected group $\mathcal{U}_{J}$ called the $J$-unitary group. We easily conclude that $W_{J}^{C}(A)$ is a connected set in the Argand's complex plane, which reduces to $W^{C}(A)$ when $r=0$ or $r=n$. In particular, denoting by $E_{i i}$ the matrix in $M_{n}$ with the $(i, i)$ entry equal to 1 and all other entries equal to 0 , we have

$$
W_{J}^{E_{i i}}(A)=\left\{\begin{aligned}
W_{J}^{+}(A), & \text { when } 1 \leq i \leq r \\
-W_{J}^{-}(A), & \text { when } r<i \leq n .
\end{aligned}\right.
$$


In general, $W^{C}(A)$ is compact for any $A, C \in M_{n}$, but it is not convex. Using Morse theory, Westwick [15] proved the convexity of $W^{C}(A)$ for any Hermitian matrix $C$ and, more recently [3], a similar convexity result was obtained for $W_{J}^{C}(A)$. Moreover, using Tarski Theorem [1], it is possible to prove that the boundary of $W_{J}^{C}(A)$ is a finite union of algebraic arcs and so a curve of class $C^{\infty}$, except for a finite number of points, for any $A, C \in M_{n}[1$, Theorem 2.4].

The main goal of this paper is to investigate the cases when the Krein space $C$-numerical range of $A$ induced by $J$ is either a singleton or a subset of the real line. In particular, we revisit and improve two well-known results on this topic.

The first result due to Bebiano et al. [1, Theorem 5.1] concerns the $(J, C)$ tracial range, when $C$ is a diagonal matrix and $J C$ has pairwise distinct main diagonal entries, and it states that $V_{J, C}(A)$ is a singleton if and only if $J A$ is a scalar matrix. A similar problem was studied in [2, Lemma 2.4] for $W_{J}^{C}(A)$, but for the case when $C$ is a non-scalar diagonal matrix and $A$ is $J$-unitarily diagonalizable. We recall that $A$ is $J$-unitarily diagonalizable if there exists $U \in \mathcal{U}_{J}$, such that $U^{[*]} A U$ is a diagonal matrix. We show that both restrictions, on the main diagonal entries of $C$ and on $A$ being $J$-unitarily diagonalizable, can be removed, and we present a more general result (Theorem 4.1).

The second result under consideration concerns the $(J, C)$-tracial range of a Hermitian matrix. Assuming that $C$ is a real diagonal matrix, $J C$ has pairwise distinct main diagonal entries and $A \in M_{n}$, it is stated in [1, Theorem 5.2] that $V_{J, C}(A)$ is a subset of the real line if and only if $A$ is Hermitian. This statement is incomplete. We find a correct criteria by showing, when $C \in M_{n}$ is a $J$-unitarily diagonalizable with real eigenvalues and $A \in M_{n}$, that $W_{J}^{C}(A)$ is a real subset if and only if one of the following conditions holds: (a) $A$ is $J$-Hermitian; (b) $\operatorname{Im}^{J} A$ is a non-zero scalar matrix and $C$ has null trace; (c) $C$ is a scalar matrix and $A$ has real trace.

In addition, we prove for $A \in M_{n}$ that the following assertions are equivalent: (a) $W_{J}(A)$ is a subset of a line; (b) $A$ is essentially $J$-Hermitian; and if $C \in$ $M_{n}$ is non-scalar $J$-unitarily diagonalizable with collinear eigenvalues, then the previous two assertions are equivalent to the following: (c) $W_{J}^{C}(A)$ is a subset of a line.

This paper is organized as follows. In Section 2, we give some preliminary results on the Krein space numerical range induced by $J$. Using the hyperbolic numerical range theorem, which characterizes the Krein space numerical range in the $2 \times 2$ case, we obtain a condition for this set to be a subset of a line. In Section 3, we give some preliminary results on the Krein space $C$-numerical range. Following analogous steps to the proof of [1, Lemma 5.1], we establish the useful reduction Lemma 3.3, which together with the characterization of $W_{J}^{C}(A)$, for particular matrices $A \in M_{n}$ in the cases $n=2$ or $n=3$, lead to some results in the paper. In Section 4 , we characterize matrices $A$ and $C$ for which the Krein space $C$-numerical range of $A$ is a singleton under some additional conditions. In Section 5, we study the case when the Krein space $C$-numerical range is a subset of a line. 


\section{Preliminary results on the Krein space numerical range}

Murnaghan [13] obtained the Elliptical Range Theorem, concerning $W(A)$ for $A \in M_{2}$, which can be stated as follows.

Theorem 2.1. Assume, $A \in M_{2}$ has eigenvalues $\alpha_{1}$ and $\alpha_{2}$. Then $W(A)$ is an elliptical disc, possibly degenerated, with foci $\alpha_{1}$ and $\alpha_{2}$, and the minor axis of the length

$$
\sqrt{\operatorname{Tr}\left(A^{*} A\right)-\left|\alpha_{1}\right|^{2}-\left|\alpha_{2}\right|^{2}}
$$

$W(A)$ has empty interior if and only if $A$ is a normal matrix.

In general, it is difficult to characterize the Krein space numerical range for an arbitrary matrix $A \in M_{n}$. However, we have the Hyperbolic Range Theorem, concerning $W_{J}(A)$ for $J=\operatorname{diag}(1,-1)$ and $A \in M_{2}$ (see [1, Theorem 3.2] and $[12$, p. 25]).

Theorem 2.2. Let $J=\operatorname{diag}(1,-1), A=\left[a_{i j}\right] \in M_{2}$ with eigenvalues $\alpha_{1}, \alpha_{2}$ and

$$
\begin{aligned}
M & =\left|\alpha_{1}\right|^{2}+\left|\alpha_{2}\right|^{2}-\operatorname{Tr}\left(A^{[*]} A\right), \\
N & =\operatorname{Tr}\left(A^{[*]} A\right)-2 \operatorname{Re}\left(\overline{\alpha_{1}} \alpha_{2}\right) .
\end{aligned}
$$

a) If $M>0$ and $N>0$, then $W_{J}(A)$ is bounded by a branch of a hyperbola with foci $\alpha_{1}$ and $\alpha_{2}$, transversal and nontransversal axis of length $\sqrt{N}$ and $\sqrt{M}$, respectively.

b) If $M>0$ and $N=0$, then consider the line l, containing $\frac{1}{2} \operatorname{Tr}(A)$, perpendicular to the line segment joining $\alpha_{1}$ and $\alpha_{2}$.

i. If $\left|a_{12}\right|=\left|a_{21}\right|$, then $W_{J}(A)$ is the line $l$.

ii. If $\left|a_{12}\right| \neq\left|a_{21}\right|$, then $W_{J}(A)$ is the complex plane except the line $l$.

c) If $M>0$ and $N<0$, then $W_{J}(A)$ is the whole complex plane.

d) If $M=0$ and $N>0$, then $W_{J}(A)$ is the line defined by $\alpha_{1}$ and $\alpha_{2}$, except the open line segment joining them.

e) If $M=N=0$, then $W_{J}(A)$ is either the singleton $\left\{\frac{1}{2} \operatorname{Tr}(A)\right\}$, when $A$ is a scalar matrix, or the line defined by $\alpha_{1}$ and $\alpha_{2}$, except $\frac{1}{2} \operatorname{Tr}(A)$, otherwise.

In the sequel, let $J=I_{r} \oplus-I_{n-r}$ for some $0 \leq r \leq n$.

If $\kappa \subset\{1, \ldots, n\}$, then $A[\kappa]$ denotes the principal submatrix of $A \in M_{n}$ that lies in rows and columns indexed by $\kappa$. When $\kappa=\{i, j\}$, we simply write $A[\kappa]=A_{i j}$. 
Lemma 2.3. For all $A \in M_{n}$ and index set $\kappa \subset\{1, \ldots, n\}$, we have

$$
W_{J[\kappa]}(A[\kappa]) \subset W_{J}(A) .
$$

Proof. Suppose $\kappa=\left\{i_{1}, \ldots, i_{k}\right\}$ with $1 \leq i_{1}<\ldots<i_{k} \leq n$ and, for simplicity of notation, let $A^{\prime}=A[\kappa]$ and $J^{\prime}=J[\kappa]$. If $z$ is an arbitrary element in $W_{J^{\prime}}\left(A^{\prime}\right)$, then there exists $x \in \mathbb{C}^{k}$ satisfying $\langle x, x\rangle_{J^{\prime}} \neq 0$ such that

$$
z=\frac{\left\langle A^{\prime} x, x\right\rangle_{J^{\prime}}}{\langle x, x\rangle_{J^{\prime}}} .
$$

We may insert zero entries into appropriate locations of $x$ to produce a vector $\widehat{x} \in \mathbb{C}^{n}$, such that $\widehat{x_{i j}}=x_{i j}, j=1, \ldots, k$, and $\widehat{x}_{j}=0$, for all the other indices $j$. Then $\langle\widehat{x}, \widehat{x}\rangle_{J} \neq 0$ and we easily check that

$$
\frac{\left\langle A^{\prime} x, x\right\rangle_{J^{\prime}}}{\langle x, x\rangle_{J^{\prime}}}=\frac{\langle A \widehat{x}, \widehat{x}\rangle_{J}}{\langle\widehat{x}, \widehat{x}\rangle_{J}},
$$

that is, $z \in W_{J}(A)$.

We say that $A$ is $J$-unitarily similar to $B$ if there exists a $J$-unitary matrix $U$, such that $A=U^{[*]} B U$. Additionally, $A$ is said to be $J$-unitarily triangularizable (diagonalizable) if $A$ is $J$-unitarily similar to a triangular (respectively, diagonal) matrix $T$; in this case, the main diagonal entries of $T$ are equal to the eigenvalues of $A$.

Theorem 2.4. Let $A \in M_{n}$. Assume that $A$ is $J$-unitarily triangularizable and $W_{J}(A)$ is a subset of a line. Then $A$ is $J$-unitarily diagonalizable.

Proof. Suppose that $A$ is not $J$-unitarily diagonalizable and $W_{J}(A)$ is a subset of a line. Without loss of generality, by property $\mathrm{P} 2$, we may consider $A$ to be in a triangular form. Hence, there exist $k<l$, such that the principal submatrix $A_{k l} \in M_{2}$ has an off-diagonal entry $b \neq 0$. By Lemma 2.3 we have

$$
W_{J_{k l}}\left(A_{k l}\right) \subset W_{J}(A) .
$$

If $J_{k l}= \pm I_{2}$, then by Theorem 2.1 the set $W_{J_{k l}}\left(A_{k l}\right)=W\left(A_{k l}\right)$ is an elliptical disc with a non-empty interior, because $A_{k l}$ is not normal. If $J_{k l}=\operatorname{diag}(1,-1)$ and $\alpha_{k}, \alpha_{l}$ are the diagonal entries of $A_{k l}$, then

$$
\begin{gathered}
M_{k l}=\left|\alpha_{k}\right|^{2}+\left|\alpha_{l}\right|^{2}-\operatorname{Tr}\left(A_{k l}^{[*]} A_{k l}\right)=|b|^{2}>0, \\
N_{k l}=\operatorname{Tr}\left(A_{k l}^{[*]} A_{k l}\right)-2 \operatorname{Re}\left(\overline{\alpha_{k}} \alpha_{l}\right)=\left|\alpha_{k}-\alpha_{l}\right|^{2}-|b|^{2},
\end{gathered}
$$

and so by Theorem 2.2 we have that the set $W_{J_{k l}}\left(A_{k l}\right)$ is either bounded by a hyperbola if $N_{k l}>0$, it is the whole complex plane if $N_{k l}<0$, or the complex plane with a removed line if $N_{k l}=0$. In any case, $W_{J}(A)$ contains a subset with a non-empty interior, a contradiction. 
If $J= \pm I_{n}$, then any matrix $A \in M_{n}$ is $J$-unitarily triangularizable, by Schur Triangularization Theorem [7, Theorem 2.3.1] and in this case the $J$-unitarily diagonalizable matrices are the normal matrices [7, Theorem 2.5.4]. Hence, we have the following corollary from Theorem 2.4.

Corollary 2.5. Let $A \in M_{n}$. If $W(A)$ is a line segment, then $A$ is a normal matrix.

\section{Preliminary results on the Krein space $C$-numerical range}

In this section, we investigate the Krein space $C$-numerical range; in particular, we revisit [1, Theorem 5.1] and [2, Lemma 2.4], concerning the case when the $(J, C)$-tracial range is a singleton, and we present in Theorem 4.1 a more general result.

The Elliptical Range Theorem, concerning $W^{C}(A)$ for $C=\operatorname{diag}\left(c_{1}, c_{2}\right) \in$ $M_{2}$ and $A \in M_{2}$, is well-known [4].

Theorem 3.1. [4] If $A$ has eigenvalues $\alpha_{1}$ and $\alpha_{2}$, then $W^{C}(A)$ is a possibly degenerated elliptical disc with foci $c_{1} \alpha_{1}+c_{2} \alpha_{2}$ and $c_{1} \alpha_{2}+c_{2} \alpha_{1}$, and minor axis of the length

$$
\left|c_{1}-c_{2}\right| \sqrt{\operatorname{Tr}\left(A^{*} A\right)-\left|\alpha_{1}\right|^{2}-\left|\alpha_{2}\right|^{2}} .
$$

On the other hand, if $J=\operatorname{diag}(1,-1)$, then the $(J, C)$-tracial range of $A \in M_{2}$ is bounded by a branch of a possibly degenerated hyperbola [1, Theorem 4.1 (i)]. For our investigation concerning the Krein space $C$-numerical range, we need the following complete characterization of the set $W_{J}^{C}(A)$ in the $2 \times 2$ case, with the degenerate occurrences explicitly included.

Theorem 3.2. Let $J=\operatorname{diag}(1,-1), C=\operatorname{diag}\left(c_{1}, c_{2}\right) \in M_{2}$ and $A=\left[a_{i j}\right] \in$ $M_{2}$ with eigenvalues $\alpha_{1}, \alpha_{2}$. Consider $z_{1}=c_{1} \alpha_{1}+c_{2} \alpha_{2}, z_{2}=c_{1} \alpha_{2}+c_{2} \alpha_{1}$, $\delta=\operatorname{Tr}(A) \operatorname{Tr}(C) / 2$ and

$$
\begin{aligned}
M & =\left|c_{1}-c_{2}\right|^{2}\left(\left|\alpha_{1}\right|^{2}+\left|\alpha_{2}\right|^{2}-\operatorname{Tr}\left(A^{[*]} A\right)\right), \\
N & =\left|c_{1}-c_{2}\right|^{2}\left(\operatorname{Tr}\left(A^{[*]} A\right)-2 \operatorname{Re}\left(\overline{\alpha_{1}} \alpha_{2}\right)\right) .
\end{aligned}
$$

a) If $M>0$ and $N>0$, then $W_{J}^{C}(A)$ is bounded by a branch of a hyperbola with foci $z_{1}$ and $z_{2}$, transversal and nontransversal axis of length $\sqrt{N}$ and $\sqrt{M}$, respectively.

b) If $M>0$ and $N=0$, then consider the line l, containing $\delta$, perpendicular to the line segment joining $z_{1}$ and $z_{2}$.

i. If $\left|a_{12}\right|=\left|a_{21}\right|$, then $W_{J}^{C}(A)$ is the line $l$.

ii. If $\left|a_{12}\right| \neq\left|a_{21}\right|$, then $W_{J}^{C}(A)$ is an open half-plane delimited by $l$. 
c) If $M>0$ and $N<0$, then $W_{J}^{C}(A)$ is the whole complex plane.

d) If $M=0$ and $N>0$, then $W_{J}^{C}(A)$ is a closed half line with endpoint $\delta$, lying on the line defined by $z_{1}$ and $z_{2}$.

e) If $M=N=0$, then $W_{J}^{C}(A)$ is either the singleton $\{\delta\}$, when $A$ or $C$ is a scalar matrix, or an open half line with the endpoint $\delta$, lying on the line defined by $z_{1}$ and $z_{2}$, otherwise.

Proof. Since $C=c_{2} I_{2}+\left(c_{1}-c_{2}\right) E_{11}$, by the properties of the Krein space $C$-numerical range, we have

$$
W_{J}^{C}(A)=c_{2} \operatorname{Tr}(A)+\left(c_{1}-c_{2}\right) W_{J}^{+}(A) .
$$

From the complete description of $W_{J}^{+}(A)[12$, p. 30], we obtain the desired result.

Given $a \in \mathbb{C}$ and a set $S \subseteq \mathbb{C}$, the notation $S+a$ designates a new set of the translations of all the elements of $S$ by $a$, namely, $S+a=\{x+a: x \in S\}$.

As usual, $S_{n}$ denotes the symmetric group of degree $n$ and $P_{\sigma}$ is the $n \times n$ permutation matrix associated with $\sigma \in S_{n}$, that is defined by $\left(P_{\sigma}\right)_{i j}=\delta_{\sigma(i) j}$. Clearly, the adjoint of $P_{\sigma}$ with respect to the inner product induced by $J$ is the matrix $P_{\sigma}^{T}$.

A useful technique in the theory of numerical ranges is to reduce problems to the $2 \times 2$ case. An efficient reduction lemma was presented in [1] for the $(J, C)$ tracial range and this together with the previous hyperbolic range theorem can lead to some interesting results. Following analogous steps to the proof of $[1$, Lemma 5.1], we can obtain the following useful generalized version.

Lemma 3.3. Let $C=\operatorname{diag}\left(c_{1}, \ldots, c_{n}\right), A=\left[a_{i j}\right] \in M_{n}, J=I_{n} \oplus-I_{n-r}$, $0 \leq r \leq n$ and $\kappa \subset\{1, \ldots, n\}$. Then

$$
W_{J^{\prime}}^{C^{\prime}}\left(A^{\prime}\right)+\sum_{i \notin \kappa} c_{i} a_{i i} \subseteq W_{J}^{C}(A),
$$

where $A^{\prime}=A[\kappa], C^{\prime}=C[\kappa]$ and $J^{\prime}=J[\kappa]$.

Proof. Let $z$ be an arbitrary point of the set $W_{J^{\prime}}^{C^{\prime}}\left(A^{\prime}\right)+\sum_{i \notin \kappa} c_{i} a_{i i}$, that is,

$$
z=\operatorname{Tr}\left(C^{\prime} J^{\prime} G^{*} J^{\prime} A^{\prime} G\right)+\sum_{i \notin \kappa} c_{i} a_{i i},
$$

where $G$ is a $J^{\prime}$-unitary matrix. Let $s$ be the cardinality of the set $\kappa$. Considering

$$
A_{1}=\left[\begin{array}{cc}
A^{\prime} & B \\
D & E
\end{array}\right], \quad C_{1}=\left[\begin{array}{cc}
C^{\prime} & O \\
O & F
\end{array}\right]
$$


and $U_{G}, P_{\sigma}$ are the matrices obtained respectively from $A, C$ and $G \oplus I_{n-s}, I_{n}$ by permuting the rows and columns $1, \ldots, s$ with the rows and columns indexed by $\kappa$, we have

$$
C_{1}\left(G \oplus I_{n-s}\right)^{[*]} A_{1}\left(G \oplus I_{n-s}\right)=\left[\begin{array}{cc}
C^{\prime} J^{\prime} G^{*} J^{\prime} A^{\prime} G & C^{\prime} J^{\prime} G^{*} J^{\prime} B \\
F D G & F E
\end{array}\right] .
$$

We remark that $P_{\sigma}$ is an orthogonal matrix. Then $P_{\sigma}^{-1}=P_{\sigma}^{T}=P_{\sigma}^{[*]}$. Simple computations yield

$$
\begin{aligned}
z & =\operatorname{Tr}\left(C^{\prime} J^{\prime} G^{*} J^{\prime} A^{\prime} G\right)+\operatorname{Tr}(F E)=\operatorname{Tr}\left(C_{1}\left(G \oplus I_{n-s}\right)^{[*]} A_{1}\left(G \oplus I_{n-s}\right)\right) \\
& =\operatorname{Tr}\left(C_{1} P_{\sigma} U_{G}^{[*]} P_{\sigma}^{T} A_{1} P_{\sigma} U_{G} P_{\sigma}^{T}\right)=\operatorname{Tr}\left(C U_{G}^{[*]} A U_{G}\right)
\end{aligned}
$$

and $U_{G} \in M_{n}$ satisfies $U_{G}^{[*]} U_{G}=I_{n}$; hence $z \in W_{J}^{C}(A)$. Then the inclusion (2) holds.

\section{Krein space $C$-numerical range as a singleton}

In [1, Theorem 5.1], it was proved that if $C$ is a diagonal matrix and $C J$ has pairwise distinct main diagonal entries, then the $(J, C)$-tracial range of $A$ is a singleton if and only if $J A$ is a scalar matrix. In [2, Lemma 2.4], a similar problem was considered for $W_{J}^{C}(A)$, but for the case when $C$ is a diagonal non-scalar matrix and $A$ is $J$-unitarily diagonalizable. Below we show that both restrictions, on the main diagonal entries of $C$ or on $A$ being $J$-unitarily diagonalizable, can be removed, so a more general result holds.

In the sequel, we adopt the following additional notations:

$$
\begin{aligned}
& S_{n}^{r}=\left\{\sigma \in S_{n}: \sigma(j)=j, j=r+1, \ldots, n\right\}, \\
& \widehat{S_{n}^{r}}=\left\{\sigma \in S_{n}: \sigma(j)=j, j=1, \ldots, r\right\},
\end{aligned}
$$

so that $S_{n}^{r}$ and $\widehat{S_{n}^{r}}$ are the sets of permutations of $\{1, \ldots, r\}$ and $\{r+1, \ldots, n\}$, respectively, when $0<r<n$. In particular, the transposition $\tau_{1}=(\mathrm{km})$ is a permutation, satisfying $\tau_{1}(k)=m, \tau_{1}(m)=k$, all the other points being fixed.

Theorem 4.1. Let $C \in M_{n}$ be a J-unitarily diagonalizable matrix and $A \in M_{n}$. Then $W_{J}^{C}(A)$ is a singleton if and only if either $A$ or $C$ is a scalar matrix.

Proof. Sufficiency. Suppose that $A$ is a scalar matrix. Then $A=\lambda I_{n}$ with $\lambda=\operatorname{Tr}(A) / n$ and

$$
W_{J}^{C}(A)=\left\{\operatorname{Tr}\left(C U^{[*]} \lambda I_{n} U\right): U^{[*]} U=I_{n}\right\}=\{\operatorname{Tr}(A) \operatorname{Tr}(C) / n\}
$$

reduces to a singleton. If $C$ is scalar, then the result follows readily from $W_{J}^{C}(A)=W_{J}^{A}(C)$. 
Necessity. Since $C$ is a $J$-unitarily diagonalizable matrix, by the property P2 of the Krein space $C$-numerical range, without loss of generality, we may consider that $C$ is a diagonal matrix.

For $0<r<n$, suppose that $A=\left[a_{i j}\right]$ and $C=\operatorname{diag}\left(c_{1}, \ldots, c_{n}\right)$ are both non-scalar matrices. So $C$ has at least two distinct main diagonal entries.

If $n=2$, then $r=1$ and so $W_{J}^{C}(A)$ is bounded by a branch of a hyperbola, possibly degenerated, but never to a singleton by Theorem 3.2.

If $n \geq 3$, then $A$ admits a non-scalar principal submatrix $A_{k l}=A[k l]$ for some $k<l$. Several possible cases appear now.

First case: Suppose there exist $k, l$ with $k \leq r<l$. It is always possible to find $m, p$ with $m \leq r<p$, such that $C[m p]$ is a non-scalar principal submatrix of $C$. (For instance, if $c_{1} \neq c_{2}$, then $c_{1} \neq c_{n}$ or $c_{2} \neq c_{n}$, and one of the submatrices $C[1 n]$ or $C[2 n]$ is non-scalar.) Let $C_{\sigma}=P_{\sigma} C P_{\sigma}^{T}$ with $\sigma=\sigma_{1} \sigma_{2}$, where $\sigma_{1}=(\mathrm{km}) \in S_{n}^{r}$ and

$\sigma_{2}=(l p) \in \widehat{S_{n}^{r}}$. Note that $P_{\sigma}$ is a $J$-unitary matrix. Thus, by the property $\mathrm{P} 2$ of the Krein space $C$-numerical range,

$$
W_{J}^{C}(A)=W_{J}^{C_{\sigma}}(A) .
$$

By Lemma 3.3, $W_{J}^{C_{\sigma}}(A)$ contains the set

$$
\Gamma_{k l}=W_{J_{k l}}^{C_{k l}^{\prime}}\left(A_{k l}\right)+\sum_{i \neq k, l} c_{\sigma(i)} a_{i i}
$$

where $C_{k l}^{\prime}=C_{\sigma}[k l]$ and $J_{k l}=\operatorname{diag}(1,-1)$. By Theorem 3.2, $W_{J_{k l}}^{C_{k l}^{\prime}}\left(A_{k l}\right)$ is bounded by a branch of a hyperbola, possibly degenerated, but never to a point, since $A_{k l}$ and $C_{k l}^{\prime}=C[m p]$ are both non-scalar matrices. Hence, $W_{J}^{C}(A)$ is not a singleton.

Second and third cases: If the first case does not hold, then either $k<l \leq r$ or $r<k<l$. Suppose $k<l \leq r$ and let $\alpha=\{1, \ldots, r\}$. The other case, $r<k<l$, may be treated similarly.

(a) If $C[\alpha]$ is not a scalar matrix, then it is possible to find $m, p$ with $m<$ $p \leq r$, such that $C[m p]$ is a non-scalar principal submatrix of $C$ and we proceed as in the first case, except that now we have $J_{k l}=I_{2}$ in the set (3). Therefore, $W_{J_{k l}}^{C_{k l}^{\prime}}\left(A_{k l}\right)$ is an elliptical disc, possibly degenerated, but never to a point, and we also conclude that $W_{J}^{C}(A)$ is not a singleton.

(b) If $C[\alpha]$ is a scalar matrix, then (under the hypothesis) there exists $j>r$, such that $C_{1}=C[k l j]$ and $A_{1}=A[k l j]$ are non-scalar principal submatrices of $C$ and $A$, respectively. By Lemma 3.3, the inclusion

$$
W_{J_{1}}^{C_{1}}\left(A_{1}\right)+\sum_{i \neq k, l, j} c_{i} a_{i i} \subseteq W_{J}^{C}(A)
$$

holds for $C_{1}=C[k l j]=\operatorname{diag}\left(c_{1}, c_{1}, c_{j}\right)$ and $J_{1}=J[k l j]=\operatorname{diag}(1,1,-1)$. Let $C_{1}^{\prime}=C_{1}-c_{1} I_{3}$. Then $C_{1}^{\prime}=\left(c_{j}-c_{1}\right) E_{33}$ with $c_{j} \neq c_{1}$. By the properties P3, $\mathrm{P} 1$ and the equality (1), we have

$$
W_{J_{1}}^{C_{1}}\left(A_{1}\right)=W_{J}^{A_{1}}\left(c_{1} I_{3}+C_{1}^{\prime}\right)=c_{1} \operatorname{Tr}\left(A_{1}\right)+W_{J_{1}}^{A_{1}}\left(C_{1}^{\prime}\right)
$$




$$
=c_{1} \operatorname{Tr}\left(A_{1}\right)+W_{J_{1}}^{C_{1}^{\prime}}\left(A_{1}\right)=c_{1} \operatorname{Tr}\left(A_{1}\right)-\left(c_{j}-c_{1}\right) W_{J_{1}}^{-}\left(A_{1}\right) .
$$

Since $A_{1}$ is non-scalar then $W_{J_{1}}^{-}\left(A_{1}\right)$ is not a singleton. Therefore, $W_{J}^{C}(A)$ is not a singleton.

The remaining cases, $r=0$ and $r=n$, follow easily from [9, Theorem 2.5].

We do not need thee assumption that $C$ is $J$-unitarily diagonalizable in the proof of the sufficiency presented above, that is, if one of the matrices $A, C \in M_{n}$ is scalar, then we have the singleton

$$
W_{J}^{C}(A)=\left\{\frac{1}{n} \operatorname{Tr}(A) \operatorname{Tr}(C)\right\} .
$$

Conjecture 4.2. Theorem 4.1 holds for any matrix $C \in M_{n}$ without the assumption that $C$ is $J$-unitarily diagonalizable.

Now we are going to show that this conjecture is true for any $A, C \in M_{2}$. To do this we need the following useful lemma. Consider $[X, Y]$ as the commutator $X Y-Y X$ for all $X, Y \in M_{n}$.

Lemma 4.3. Let $A, C \in M_{n}$ be such that $W_{J}^{C}(A)$ is a singleton. Then $U^{[*]} A U$ commutes with $C$ for any $U \in \mathcal{U}_{J}$.

Proof. Let $U \in \mathcal{U}_{J}$ and for simplicity of notation write $A_{U}=U^{[*]} A U$. Let $S \in M_{n}$ be a Hermitian matrix and $t \in \mathbb{R}$ in a neighborhood of 0 . Then the matrix $V_{S, t}=e^{i t J^{1 / 2} S J^{1 / 2}}$ is $J$-unitary and the differentiable function

$$
f(t)=\operatorname{Tr}\left(C V_{S, t}^{[*]} A_{U} V_{S, t}\right)
$$

is constant, because $W_{J}^{C}\left(A_{U}\right)=W_{J}^{C}(A)$ is a singleton, and so $f^{\prime}(t)=0$. Since

$$
f^{\prime}(0)=i \operatorname{Tr}\left(S J^{1 / 2}\left[C, A_{U}\right] J^{1 / 2}\right),
$$

from $f^{\prime}(0)=0$ for any Hermitian matrix $S$, we find that $\operatorname{Tr}\left(X J^{1 / 2}\left[C, A_{U}\right] J^{1 / 2}\right)=$ 0 for any $X \in M_{n}$. This implies that $J^{1 / 2}\left[C, A_{U}\right] J^{1 / 2}=0$ or, equivalently, $A_{U} C=C A_{U}$.

Theorem 4.4. Let $A, C \in M_{2}$. Then $W_{J}^{C}(A)$ is a singleton if and only if either $A$ or $C$ is a scalar matrix.

Proof. If $J= \pm I_{2}$, then the result is clearly true. Let $J=\operatorname{diag}(1,-1)$. If $A=\left[a_{i j}\right] \in M_{2}$, then there exists a $J$-unitary matrix $U \in M_{2}$, such that

$$
U^{[*]} A U=\frac{1}{2} \operatorname{Tr}(A) I_{2}+\beta_{k} A_{k}
$$

where

$$
A_{k}=\left[\begin{array}{cc}
k & a_{k} e^{i \theta_{k}} \\
b_{k} e^{i \theta_{k}} & -k
\end{array}\right], \quad \beta_{k}=\frac{1}{2} \operatorname{Tr}(A J) k+1-k
$$


with $k=0$ if $a_{11}=a_{22}$ and $k=1$ otherwise, such that

$$
a_{k}=\left|a_{12}\right| /\left|\beta_{k}\right|, \quad b_{k}=\left|a_{21}\right| /\left|\beta_{k}\right|, \quad \theta_{k}=\frac{1}{2} \arg \left(a_{12} a_{21}\right)-\arg \beta_{k} .
$$

(See proof of [12, Theorem 1.27], for more details.) Then

$$
W_{J}^{C}(A)=\frac{1}{2} \operatorname{Tr}(A) \operatorname{Tr}(C)+\beta_{k} W_{J}^{C}\left(A_{k}\right)
$$

In an analogous way, for $C=\left[c_{i j}\right] \in M_{2}$, there exists a $J$-unitary matrix $V \in M_{2}$, such that

$$
V^{[*]} C V=\frac{1}{2} \operatorname{Tr}(C) I_{2}+\gamma_{p} C_{p}
$$

where

$$
C_{p}=\left[\begin{array}{cc}
p & c_{p} e^{i \eta_{p}} \\
d_{p} e^{i \eta_{p}} & -p
\end{array}\right], \quad \gamma_{p}=\frac{1}{2} \operatorname{Tr}(C J) p+1-p .
$$

with $p=0$ if $c_{11}=c_{22}$ and $p=1$ otherwise, such that

$$
c_{p}=\left|c_{12}\right| /\left|\gamma_{p}\right|, \quad d_{p}=\left|c_{21}\right| /\left|\gamma_{p}\right|, \quad \eta_{p}=\frac{1}{2} \arg \left(c_{12} c_{21}\right)-\arg \gamma_{p} .
$$

So

$$
W_{J}^{C}\left(A_{k}\right)=W_{J}^{A_{k}}(C)=\frac{1}{2} \operatorname{Tr}\left(A_{k}\right) \operatorname{Tr}(C)+\gamma_{p} W_{J}^{A_{k}}\left(C_{p}\right)=\gamma_{p} W_{J}^{A_{k}}\left(C_{p}\right),
$$

since $\operatorname{Tr}\left(A_{k}\right)=0$. Combining (4) and (5), we have

$$
W_{J}^{C}(A)=\frac{1}{2} \operatorname{Tr}(A) \operatorname{Tr}(C)+\beta_{k} \gamma_{p} W_{J}^{C_{p}}\left(A_{k}\right) .
$$

The general situation splits now into the following four cases.

First case: Suppose $a_{11}=a_{22}$ and $c_{11}=c_{22}$. Then $k=p=0$ so that $\beta_{0}=\gamma_{0}=1$, that is,

$$
W_{J}^{C}(A)=\frac{1}{2} \operatorname{Tr}(A) \operatorname{Tr}(C)+W_{J}^{C_{0}}\left(A_{0}\right)=\frac{1}{2} \operatorname{Tr}(A) \operatorname{Tr}(C)+e^{i\left(\theta_{0}+\eta_{0}\right)} W_{J}^{\bar{C}}(\bar{A})
$$

where

$$
\bar{A}=\left[\begin{array}{ll}
0 & a \\
b & 0
\end{array}\right], \quad \bar{C}=\left[\begin{array}{ll}
0 & c \\
d & 0
\end{array}\right] .
$$

and $a=a_{0}, b=b_{0}, c=c_{0}, d=d_{0}$ for the simplicity of notations. If $W_{J}^{C}(A)$ is a singleton, then $W_{J}^{\bar{C}}(\bar{A})$ is a singleton. Observe that

$$
Q=\left[\begin{array}{ll}
u & v \\
v & u
\end{array}\right]
$$

with $u, v \in \mathbb{R}$ such that $u^{2}-v^{2}=1$, is a $J$-unitary matrix. By Theorem 4.3, $Q^{[*]} \bar{A} Q$ commutes with $\bar{C}$. Therefore the main diagonal entries of $\left[Q^{[*]} \bar{A} Q, \bar{C}\right]$ are zero, this implying

$$
\left(b u^{2}-a v^{2}\right) c-\left(a u^{2}-b v^{2}\right) d=0 .
$$


Having in mind that $u^{2}=1+v^{2}$, we find

$$
b c-a d+(b-a)(c+d) v^{2}=0
$$

for any $v$. Hence $b c-a d=0$ and either $a=b$ or $c+d=0$. Then one of the following holds: i. $a=b=0$ or ii. $a=b$ and $c=d$ or iii. $c=d=0$.

If ii. holds, considering the matrices $R_{t}=\operatorname{diag}\left(e^{i t}, e^{-i t}\right), t \in \mathbb{R}$, which are $J$-unitary, we find $\operatorname{Tr}\left(\bar{C} R_{t}^{[*]} \bar{A} R_{t}\right)=2 a c \cos (2 t)$. Since $W_{J}^{\bar{C}}(\bar{A})$ is a singleton, then we have

$$
2 a c=\operatorname{Tr}\left(\bar{C} R_{0}^{[*]} \bar{A} R_{0}\right)=\operatorname{Tr}\left(\bar{C} R_{\pi / 4}^{[*]} \bar{A} R_{\pi / 4}\right)=0
$$

and so either i. or iii. holds. Hence, either $\bar{A}=O$ or $\bar{C}=O$. Therefore, one of the matrices $A, C$ is scalar.

Second case: Suppose $a_{11}=a_{22}$ and $c_{11} \neq c_{22}$. Then $k=0$ and $p=1$ so that $\beta_{0}=1$ and $\gamma_{1}=\frac{1}{2} \operatorname{Tr}(C J) \neq 0$, that is,

$$
W_{J}^{C}(A)=\frac{1}{2} \operatorname{Tr}(A) \operatorname{Tr}(C)+\gamma_{1} W_{J}^{C_{1}}\left(A_{0}\right)=\frac{1}{2} \operatorname{Tr}(A) \operatorname{Tr}(C)+\gamma_{1} e^{i \theta_{0}} W_{J}^{\tilde{C}}(\bar{A})
$$

where

$$
\bar{A}=\left[\begin{array}{ll}
0 & a \\
b & 0
\end{array}\right], \quad \tilde{C}=\left[\begin{array}{cc}
1 & c e^{i \eta} \\
d e^{i \eta} & -1
\end{array}\right] .
$$

and $a=a_{0}, b=b_{0}, c=c_{1}, d=d_{1}, \eta=\eta_{1}$ for simplicity of notation. If $W_{J}^{C}(A)$ is a singleton, then $W_{J}^{\tilde{C}}(\bar{A})$ is a singleton. Hence $\bar{A}$ commutes with $\tilde{C}$. This implies $a=b=0$. Then $A$ is a scalar matrix.

Third case: If $a_{11} \neq a_{22}$ and $c_{11}=c_{22}$, then we may reduce to the second case by interchanging the roles of $A$ and $C$, having in mind that $W_{J}^{C}(A)=W_{J}^{A}(C)$.

Fourth case: Suppose $a_{11} \neq a_{22}$ and $c_{11} \neq c_{22}$. Then $k=p=1$ so that $\beta_{1}=\frac{1}{2} \operatorname{Tr}(A J) \neq 0$ and $\gamma_{1}=\frac{1}{2} \operatorname{Tr}(C J) \neq 0$, that is,

$$
W_{J}^{C}(A)=\frac{1}{2} \operatorname{Tr}(A) \operatorname{Tr}(C)+\beta_{1} \gamma_{1} W_{J}^{C_{1}}\left(A_{1}\right)=\frac{1}{2} \operatorname{Tr}(A) \operatorname{Tr}(C)+\beta_{1} \gamma_{1} W_{J}^{\bar{C}}(\bar{A})
$$

where

$$
\tilde{A}=\left[\begin{array}{cc}
1 & a e^{i \theta} \\
b e^{i \theta} & -1
\end{array}\right], \quad \tilde{C}=\left[\begin{array}{cc}
1 & c e^{i \eta} \\
d e^{i \eta} & -1
\end{array}\right] .
$$

and $a=a_{1}, b=b_{1}, c=c_{1}, d=d_{1}, \theta=\theta_{1}, \eta=\eta_{1}$ for the simplicity of notations. If $W_{J}^{C}(A)$ is a singleton, then $W_{J}^{\tilde{C}}(\tilde{A})$ is a singleton. Hence $\tilde{A}$ commutes with $\tilde{C}$. Now direct computations imply that $\tilde{A}=\tilde{C}$. But

$$
\operatorname{Tr}\left(\tilde{A} Q^{[*]} \tilde{A} Q\right)=2+4 v^{2}+e^{2 i \theta}\left(2 a b-(a-b)^{2} v^{2}\right)
$$

is not constant, that is, $W_{J}^{\tilde{A}}(\tilde{A})$ is not a singleton, a contradiction. 


\section{Krein space $C$-numerical range as a subset of a line}

We know that $W_{J}^{+}(A)$ is the empty set when $J=-I_{n}$; otherwise, $W_{J}^{+}(A)$ is a subset of the real line if and only if $A$ is $J$-Hermitian, as already stated. However, we can not replace $W_{J}^{+}(A)$ by $W_{J}^{C}(A)$ in the previous statement, even if the matrix $C$ is assumed real diagonal, as the following easy counterexamples show.

Example 5.1. Let $J=\operatorname{diag}(1,-1), A=i J$ and $C=I_{2}$. Since $C$ is a scalar matrix and $A$ has null trace, then $W_{J}^{C}(A)=\{0\} \subset \mathbb{R}$ and $A$ is not $J$-Hermitian.

Example 5.2. Let $J=C=\operatorname{diag}(1,-1)$ and $A=\operatorname{diag}(1+3 i,-2+3 i)$. Then

$$
\operatorname{Tr}\left(A^{[*]} A\right)=|1+3 i|^{2}+|-2+3 i|^{2}=23 .
$$

Hence, $M=0$ and simple computations show that

$$
N=2(23-2 \times 7)=18>0 .
$$

By Theorem 3.2, $W_{J}^{C}(A)=[3,+\infty) \subset \mathbb{R}$ and $A$ is not $J$-Hermitian.

We recall that for $A \in M_{n}$, we may write $A=\operatorname{Re}^{J} A+i \operatorname{Im}^{J} A$, where

$$
\operatorname{Re}^{J} A=\frac{1}{2}\left(A+A^{[*]}\right) \quad \text { and } \quad \operatorname{Im}^{J} A=\frac{1}{2 i}\left(A-A^{[*]}\right)
$$

are $J$-Hermitian matrices. If $J=I_{n}$, we simply write $A=\operatorname{Re} A+i \operatorname{Im} A$, which is the Cartesian decomposition of $A$.

Further, when $S$ is a subset of the complex plane, the projections of $S$ onto the real and imaginary axis are denoted as usual by $\operatorname{Re} S$ and $\operatorname{Im} S$, respectively.

Lemma 5.3. For $A, C \in M_{n}$ the projections of $W_{J}^{C}(A)$ onto the real and imaginary axis are

$\left.\operatorname{Re} W_{J}^{C}(A)=\left\{\operatorname{Tr}\left(\operatorname{Re}^{J} C U^{[*]} \operatorname{Re}^{J} A U\right)-\operatorname{Tr}\left(\operatorname{Im}^{J} C U^{[*]} \operatorname{Im}^{J} A U\right)\right): U \in \mathcal{U}_{J}\right\}$,

$\operatorname{Im} W_{J}^{C}(A)=\left\{\operatorname{Tr}\left(\operatorname{Re}^{J} C U^{[*]} \operatorname{Im}^{J} A U\right)+\operatorname{Tr}\left(\operatorname{Im}^{J} C U^{[*]} \operatorname{Re}^{J} A U\right): U \in \mathcal{U}_{J}\right\}$, respectively. If $C$ is $J$-Hermitian, then

$$
\operatorname{Re} W_{J}^{C}(A)=W_{J}^{C}\left(\operatorname{Re}^{J} A\right) \quad \text { and } \quad \operatorname{Im} W_{J}^{C}(A)=W_{J}^{C}\left(\operatorname{Im}^{J} A\right) .
$$

Proof. If $z \in W_{J}^{C}(A)$, then there exists a $J$-unitary matrix $U$, such that $z=\operatorname{Tr}\left(C U^{[*]} A U\right)$ and we get

$$
2 \operatorname{Re} z=\operatorname{Tr}\left(C U^{[*]} A U\right)+\operatorname{Tr}\left(C^{[*]} U^{[*]} A^{[*]} U\right) .
$$


From $A^{[*]}=\operatorname{Re}^{J} A-i \operatorname{Im}^{J} A$ and $C^{[*]}=\operatorname{Re}^{J} C-i \operatorname{Im}^{J} C$, easy computations yield

$$
\operatorname{Re} z=\operatorname{Tr}\left(\operatorname{Re}^{J} C U^{[*]} \operatorname{Re}^{J} A U\right)-\operatorname{Tr}\left(\operatorname{Im}^{J} C U^{[*]} \operatorname{Im}^{J} A U\right)
$$

and the projection of $W_{J}^{C}(A)$ onto the real axis is the set (6). On the other hand,

$\bar{z}=\operatorname{Tr}\left(C^{[*]} U^{[*]} A^{[*]} U\right)=z-2 i \operatorname{Tr}\left(\operatorname{Re}^{J} C U^{[*]} \operatorname{Im}^{J} A U+\operatorname{Im}^{J} C U^{[*]} \operatorname{Re}^{J} A U\right)$.

so the projection of $W_{J}^{C}(A)$ onto the imaginary axis is given by the set of all the elements

$$
\operatorname{Tr}\left(\operatorname{Re}^{J} C U^{[*]} \operatorname{Im}^{J} A U\right)+\operatorname{Tr}\left(\operatorname{Im}^{J} C U^{[*]} \operatorname{Re}^{J} A U\right)
$$

for any $U \in \mathcal{U}_{J}$. In particular, if $C$ is $J$-Hermitian, then $\operatorname{Re}^{J} C=C, \operatorname{Im}^{J} C=O$ and we easily have

$$
\begin{aligned}
& \operatorname{Re} W_{J}^{C}(A)=\left\{\operatorname{Tr}\left(C U^{[*]} \operatorname{Re}^{J} A U\right): U \in \mathcal{U}_{J}\right\}=W_{J}^{C}\left(\operatorname{Re}^{J} A\right), \\
& \operatorname{Im} W_{J}^{C}(A)=\left\{\operatorname{Tr}\left(C U^{[*]} \operatorname{Im}^{J} A U\right): U \in \mathcal{U}_{J}\right\}=W_{J}^{C}\left(\operatorname{Im}^{J} A\right),
\end{aligned}
$$

that is, (7) holds.

Next, assuming $C$ is $J$-unitarily diagonalizable with real eigenvalues, we present a correct characterization of the matrices $A, C$ for which $W_{J}^{C}(A)$ is a subset of the real line. The statement presented in a previous attempt made in $[1$, Theorem 5.2] for the $(J, C)$-tracial range, assuming that the matrix $J C$ has pairwise distinct main diagonal entries, is incomplete (as well as the statement in [11], corresponding to the case $J=I_{n}$, as can be seen in [5]). Now, we correct the above result.

Theorem 5.4. Let $C \in M_{n}$ be J-unitarily diagonalizable with real eigenvalues and $A \in M_{n}$. Then $W_{J}^{C}(A)$ is a subset of the real line if and only if one of the following conditions holds:

(a) A is J-Hermitian;

(b) $\operatorname{Im}^{J} A$ is a non-zero scalar matrix and $C$ has null trace;

(c) $C$ is a scalar matrix and $A$ has real trace.

Proof. Since $C$ is $J$-unitarily diagonalizable with real eigenvalues, then $C$ is $J$-Hermitian and by Lemma 5.3 we have

$$
\operatorname{Im} W_{J}^{C}(A)=W_{J}^{C}\left(\operatorname{Im}^{J} A\right) .
$$

It is clear that $W_{J}^{C}(A)$ is a subset of the real line if and only if $\operatorname{Im} W_{J}^{C}(A)=\{0\}$. It follows from Theorem 4.1 that one of the matrices $\operatorname{Im}^{J} A$ or $C$ is scalar. In that case,

$$
W_{J}^{C}\left(\operatorname{Im}^{J} A\right)=\left\{\frac{1}{n} \operatorname{Tr}\left(\operatorname{Im}^{J} A\right) \operatorname{Tr}(C)\right\}=\{0\} .
$$


Firstly, suppose $\operatorname{Im}^{J} A$ is scalar. If $\operatorname{Im}^{J} A=O$, then $A$ is $J$-Hermitian and (a) holds. Otherwise, $\operatorname{Im}^{J} A$ is a non-zero scalar matrix (with non-zero trace) and we conclude from (8) that $\operatorname{Tr}(C)=0$, that is, (b) holds. Finally, if $C$ is a scalar matrix, then $W_{J}^{C}(A)$ reduces to the real number $\frac{1}{n} \operatorname{Tr}(A) \operatorname{Tr}(C)$ and since $C$ has real trace, then the trace of $A$ must be real too.

$(\Leftarrow)$ This implication is trivially verified.

Next, we find some conditions under which the projection of the Krein space $C$-numerical range of $A$ onto the real or imaginary axis is a singleton.

Proposition 5.5. Let $A, C \in M_{n}$ be such that the set $\left\{\operatorname{Re}^{J} A, \operatorname{Re}^{J} C, \operatorname{Im}^{J} A, \operatorname{Im}^{J} C\right\}$ contains a pair of scalar matrices. Then $W_{J}^{C}(A)$ is contained in a line parallel to the real or imaginary axis, passing through the point $\frac{1}{n} \operatorname{Tr}(A) \operatorname{Tr}(C)$.

Proof. Under the hypothesis, one of the following conditions holds:

i. at least one of the matrices $A, C$ is scalar;

ii. either $\operatorname{Re}^{J} A$ and $\operatorname{Re}^{J} C$ or $\operatorname{Im}^{J} A$ and $\operatorname{Im}^{J} C$ are scalar matrices;

iii. either $\operatorname{Re}^{J} A$ and $\operatorname{Im}^{J} C$ or $\operatorname{Im}^{J} A$ and $\operatorname{Re}^{J} C$ are scalar matrices;

When $X \in M_{n}$ is scalar and $Y \in M_{n}$, it is clear that $X=\frac{1}{n} \operatorname{Tr}(X) I_{n}$ and by the cyclical property of the trace, we have

$$
\operatorname{Tr}\left(X U^{[*]} Y U\right)=\operatorname{Tr}\left(Y U X U^{[*]}\right)=\frac{1}{n} \operatorname{Tr}(X) \operatorname{Tr}(Y)
$$

for all $U \in \mathcal{U}_{J}$.

If i. holds, than $W_{C}^{J}(A)=\left\{\frac{1}{n} \operatorname{Tr}(A) \operatorname{Tr}(C)\right\}$ and the result follows.

If ii. holds, by Lemma 5.3, it follows that the projection of $W_{J}^{C}(A)$ onto the imaginary axis is the singleton:

$$
\begin{aligned}
\operatorname{Im} W_{J}^{C}(A) & =\left\{\frac{1}{n} \operatorname{Tr}\left(\operatorname{Re}^{J} C\right) \operatorname{Tr}\left(\operatorname{Im}^{J} A\right)+\frac{1}{n} \operatorname{Tr}\left(\operatorname{Im}^{J} C\right) \operatorname{Tr}\left(\operatorname{Re}^{J} A\right)\right\} \\
& =\left\{\frac{1}{n} \operatorname{Im}(\operatorname{Tr}(A) \operatorname{Tr}(C))\right\} .
\end{aligned}
$$

Hence, $W_{J}^{C}(A)$ is contained in a line parallel to the real axis, passing through point $\frac{1}{n} \operatorname{Tr}(A) \operatorname{Tr}(C)$.

If iii. holds, then by Lemma 5.3 we have that the projection of $W_{J}^{C}(A)$ onto the real axis reduces to the singleton:

$$
\begin{aligned}
\operatorname{Re} W_{J}^{C}(A) & =\left\{\frac{1}{n} \operatorname{Tr}\left(\operatorname{Re}^{J} C\right) \operatorname{Tr}\left(\operatorname{Re}^{J} A\right)-\frac{1}{n} \operatorname{Tr}\left(\operatorname{Im}^{J} C\right) \operatorname{Tr}\left(\operatorname{Im}^{J} A\right)\right\} \\
& =\left\{\frac{1}{n} \operatorname{Re}(\operatorname{Tr}(A) \operatorname{Tr}(C))\right\},
\end{aligned}
$$

that is, the set $W_{J}^{C}(A)$ is contained in a line parallel to the imaginary axis, passing through the point $\frac{1}{n} \operatorname{Tr}(A) \operatorname{Tr}(C)$. 
Definition 5.6. A matrix $A \in M_{n}$ is called essentially $J$-Hermitian if there exist $\alpha, \beta \in \mathbb{C}$ with $\beta \neq 0$, such that $\alpha I_{n}+\beta A$ is $J$-Hermitian.

Theorem 5.7. Let $A \in M_{n}$. Then the following assertions are equivalent:

(a) $W_{J}(A)$ is a subset of a line;

(b) A is essentially J-Hermitian.

Further, if $C \in M_{n}$ is non-scalar $J$-unitarily diagonalizable with collinear eigenvalues, then the previous two assertions are equivalent to the following:

(c) $W_{J}^{C}(A)$ is a subset of a line.

Proof. (a) $\Rightarrow$ (b) Suppose that $W_{J}(A)$ is a subset of a line. After an appropriate translation and rotation, we find that $W_{J}\left(\alpha I_{n}+e^{i \phi} A\right) \subset \mathbb{R}$ for some $\alpha \in \mathbb{C}$ and $\phi \in[0,2 \pi)$. By $(\mathrm{C} 2)$ this is equivalent to the assertion that $\alpha I_{n}+\beta A$ is $J$ Hermitian, with $\beta=e^{i \phi}$, that is, (b) holds.

If $C \in M_{n}$ is non-scalar $J$-unitarily diagonalizable with collinear eigenvalues, then $C$ is essentially $J$-Hermitian, so that there exist $\gamma \in \mathbb{C}$ and $\psi \in[0,2 \pi)$, such that

$$
D=\gamma I_{n}+e^{i \psi} C
$$

is a non-scalar $J$-unitarily diagonalizable matrix with real eigenvalues.

(b) $\Rightarrow$ (c) Suppose that $\alpha I_{n}+\beta A$ is $J$-Hermitian for some $\alpha, \beta \in \mathbb{C}$ with $\beta \neq 0$. Since $D$ is a $J$-Hermitian matrix too, then $W_{J}^{D}\left(\alpha I_{n}+\beta A\right)$ is contained in the real line. By Properties P1-P3 and recalling (9), we get

$W_{J}^{D}\left(\alpha I_{n}+\beta A\right)=\alpha \operatorname{Tr}(D)+\beta W_{J}^{A}(D) \quad$ and $\quad W_{J}^{A}(D)=\gamma \operatorname{Tr}(A)+e^{i \psi} W_{J}^{C}(A)$.

Therefore, we may conclude that $W_{J}^{C}(A)$ is a subset of a line.

(c) $\Rightarrow$ (a) Suppose that $W_{J}^{C}(A)$ is a subset of a line $L$. Then $W_{J}^{A}(C) \subseteq L$, by property $\mathrm{P} 1$. Therefore, after an appropriate translation and rotation, we find $\alpha \in \mathbb{C}$ and $\phi \in[0,2 \pi)$, such that $W_{J}^{D}\left(\alpha I_{n}+e^{i \phi} A\right)$ is contained in the real line, with $D$ given by (9). By Theorem 5.4, we may conclude that $\operatorname{Im}^{J}\left(\alpha I_{n}+e^{i \phi} A\right)$ is a scalar matrix. It follows that

$$
W_{J}\left(\alpha I_{n}+e^{i \phi} A\right)=\alpha+e^{i \phi} W_{J}(A)
$$

is contained in a line (parallel to the real axis) and then $W_{J}(A)$ is a subset of a line.

At last, we extend the result of Theorem 2.4 to the Krein space $C$-numerical range, when $C$ is a non-scalar $J$-unitarily diagonalizable matrix.

Theorem 5.8. Let $C \in M_{n}$ be non-scalar $J$-unitarily diagonalizable and $A \in$ $M_{n}$ be $J$-unitarily triangularizable. If $W_{J}^{C}(A)$ is a subset of a line, then $A$ is $J$-unitarily diagonalizable. 
Proof. Suppose that $A$ is not $J$-unitarily diagonalizable and $W_{J}^{C}(A)$ is a subset of a line. Without loss of generality, by property $\mathrm{P} 2$, we may consider that $C=\operatorname{diag}\left(c_{1}, \ldots, c_{n}\right), A$ is a triangular matrix and there exist $k<l$, such that the principal submatrix $A_{k l}=A[k l]$ has a off-diagonal entry $b \neq 0$.

If $r=0$ or $r=n$, then $W_{J}^{C}(A)=W_{C}(A)$. Since $A$ is not normal, then by [9, Lemma 2.4] the set $W_{C}(A)$ contains a nondegenerated elliptical disc, a contradiction.

Next, assume $0<r<n$. Firstly, if $n=2$, then $r=1$. Assuming that the eigenvalues of $A$ are $\alpha_{1}, \alpha_{2}$, then we have $M=|b|^{2}>0$ and $N=\left|\alpha_{1}-\alpha_{2}\right|^{2}-|b|^{2}$ considered at the Hyperbolic Range Theorem, which allow us to conclude that $W_{J}^{C}(A)$ is an open half-plane if $N=0$, the whole complex plane if $N<0$ or a branch of a hyperbola (with interior) if $N>0$. In any case, $W_{J}^{C}(A)$ is not a subset of a line. Finally, if $n \geq 3$, then divide the proof into two cases.

First case: If $k \leq r<l$, it is possible to find $m, p$ with $m \leq r<p$ such that $C[m p]$ is a non-scalar principal submatrix of $C$. Let $C_{\sigma}=P_{\sigma} C P_{\sigma}^{T}$ with $\sigma=\sigma_{1} \sigma_{2}$, where $\sigma_{1}=(\mathrm{km}) \in S_{n}^{r}$ and $\sigma_{2}=(l p) \in S_{n}^{r}$. As in the proof of Theorem 4.1, $P_{\sigma}$ is $J$-unitary and $W_{J}^{C}(A)=W_{C_{\sigma}}^{J}(A)$. By Lemma 3.3, $W_{C_{\sigma}}^{J}(A)$ contains

$$
\Gamma_{k l}=W_{C_{k l}^{\prime}}^{J_{k l}}\left(A_{k l}\right)+\sum_{i \neq k, l} c_{\sigma(i)} a_{i i},
$$

with $C_{k l}^{\prime}=C_{\sigma}[k l]$ and $J_{k l}=\operatorname{diag}(1,-1)$. Let

$M_{k l}=\left|c_{\sigma(k)}-c_{\sigma(l)}\right|^{2}|b|^{2} \quad$ and $\quad N_{k l}=\left|c_{\sigma(k)}-c_{\sigma(l)}\right|^{2}\left(\left|a_{k k}-a_{l l}\right|^{2}-|b|^{2} \mid\right)$.

Since $M_{k l}>0$, then by Theorem 3.2 we find that $W_{C_{k l}^{\prime}}^{J_{k l}}\left(A_{k l}\right)$ is an open half plane if $N_{k l}=0$, the whole complex plane if $N_{k l}<0$ or a branch of a hyperbola (with interior) if $N_{k l}>0$. Therefore, $W_{J}^{C}(A)$ is not a subset of a line.

Second case: If the first case does not hold, then either $k<l \leq r$ or $r<k<l$. Suppose $k<l \leq r$ and let $\alpha=\{1, \ldots, r\}$. The case $r<k<l$ may be treated similarly.

(a) If $C[\alpha]$ is not a scalar matrix, then it is possible to find $m, p$ with $m<$ $p \leq r$, such that $C[m p]$ is a non-scalar principal submatrix of $C$ and we proceed as in the first case, except that the inclusion of the set $\Gamma_{k l}$ in $W_{J}^{C_{\sigma}}(A)$ holds with $J_{k l}=I_{2}$. Therefore, $W_{J_{k l}}^{C_{k l}^{\prime}}\left(A_{k l}\right)$ is a non-degenerated elliptical disc, since $A_{k l}$ is non-normal and $C_{k l}^{\prime}$ is non-scalar. It follows that $\Gamma_{k l}$ is a non-degenerated elliptical disc contained in $W_{J}^{C}(A)$.

(b) If $C[\alpha]$ is a scalar matrix, then (under the hypothesis) there exists $j>r$, such that $A_{1}=A[k l j]$ is a non-diagonal principal submatrix of $A$. If $A$ is assumed to be upper triangular, then

$$
A_{1}=\left[\begin{array}{ccc}
a_{k k} & b & 0 \\
0 & a_{l l} & 0 \\
0 & 0 & a_{j j}
\end{array}\right] .
$$

By Lemma 3.3, the inclusion

$$
W_{C_{1}}^{J_{1}}\left(A_{1}\right)+\sum_{i \neq k, l, j} c_{i} a_{i i} \subset W_{J}^{C}(A)
$$


holds for $C_{1}=C[k l j]=\operatorname{diag}\left(c_{1}, c_{1}, c_{j}\right)$ and $J_{1}=J[k l j]=\operatorname{diag}(1,1,-1)$. Let $C_{1}^{\prime}=C_{1}-c_{1} I_{3}$. Then $C_{1}^{\prime}=\left(c_{j}-c_{1}\right) E_{33}$ with $c_{j} \neq c_{1}$. As in the proof of Theorem 4.1, we have

$$
W_{C_{1}}^{J_{1}}\left(A_{1}\right)=c_{1} \operatorname{Tr}\left(A_{1}\right)-\left(c_{j}-c_{1}\right) W_{J_{1}}^{-}\left(A_{1}\right)
$$

It is clear that $-W_{J_{1}}^{-}\left(A_{1}\right)$ contains the point $a_{j j}$ and $W_{J_{1}}^{+}\left(A_{1}\right)$ contains $W\left(A_{k l}\right)$ which is the elliptical disc with foci $a_{k k}$ and $a_{l l}$ (a circular disc if $a_{k k}=a_{l l}$ ) with minor axis of length $|b| \neq 0$. Then $W_{J_{1}}\left(A_{1}\right)$ is the pseudo-convex hull of the point $a_{j j}$ and the elliptical disc $W\left(A_{k l}\right)$ constructed as follows: for each $z \in W\left(A_{k l}\right)$, all the points of the disjoint union of the closed semi-lines with endpoints $z$ and $a_{j j}$, and support line containing the line segment joining $z$ and $a_{j j}$, belong to $W_{J_{1}}\left(A_{1}\right)$. If $a_{j j} \in W\left(A_{k l}\right)$, then $W_{J_{1}}\left(A_{1}\right)$ is the whole complex plane. In any case, $-W_{J_{1}}^{-}\left(A_{1}\right)$ contains a subset of the complex plane with non-empty interior. Therefore, $W_{J}^{C}(A)$ is not contained in a line.

As a consequence, we obtain the following corollary.

Corollary 5.9. Let $C \in M_{n}$ be a non-scalar normal matrix and $A \in M_{n}$. If $W^{C}(A)$ is a subset of a line, then $A$ is a normal matrix.

\section{Acknowledgements}

The work of the first author is supported by the grant RFBR 16-01-00113. The work of the second author was supported by Portuguese funds through the Center for Research and Development in Mathematics and Applications (CIDMA) and the Portuguese Foundation for Science and Technology (FCT Fundação para a Ciência e a Tecnologia), within the project UID/MAT/0416/2013. The work of the third author was financed by Portuguese Funds through FCT Fundação para a Ciência e Tecnologia, within the Project UID/MAT/00013/2013.

[1] N. Bebiano, R. Lemos, J. da Providência and G. Soares, On generalized numerical ranges of operators on an indefinite inner product space, Linear Multilinear Algebra 52 (3-4), (2004), 203-233.

[2] N. Bebiano, R. Lemos, J. da Providência and G. Soares, Inequalities for $J$-Hermitian matrices, Linear Algebra Appl. 407 (2005), 125-139.

[3] N. Bebiano, H. Nakazato and J. da Providência, Convexity of the Krein space tracial range and Morse theory, C. R. Math. Rep. Acad. Sci. Canada 32 (4) 2010, 97-105.

[4] M. Goldberg, E.G. Straus, Elementary inclusion relations for generalized numerical ranges, Linear Algebra Appl. 18 (1977), 1-24.

[5] A. Guterman, R. Lemos and G. Soares, On the $C$-determinantal range for special classes of matrices, Applied Mathematics and Computation, 275 (2016), 86-94. 
[6] F. Hausdorff, Der Wertvorrat einer Bilinearform, Math. Z. 3 (1919), 314-316.

[7] R.A. Horn and C.R. Johnson, Matrix analysis, Cambridge University Press, Cambridge, 1985.

[8] D.W. Kribs, A. Pasieka, M. Laforest, C. Ryan, M.P. da Silva, Research problems on numerical ranges in quantum computing, Linear and Multilin. Algebra, 57, no.5 (2009), 491-502.

[9] C.-K. Li, The C-convex matrices, Linear Multilin. Algebra 21 (1987), 303-312.

[10] C.-K. Li, N.-K. Tsing and F. Uhlig, Numerical ranges of an operator in an indefinite inner product space, Electr. J. Linear Algebra 1 (1996), $1-17$.

[11] C.-K. Li, $C$-numerical ranges and $C$-numerical radii, Linear Multilin. Algebra 37 (1994), 51-82.

[12] R. Lemos, Contradomínio Numérico, Desigualdades Matriciais e suas Aplicações em Física, Ph.D thesis, University of Aveiro, 2005.

[13] F. D. Murnaghan, On the field of values of a square matrix, Proc. Nat. Acad. Sci. 18 (1932), 246-248.

[14] O. Toeplitz, Das algebraische Analogon zu einem Satz von Fejer, Math. Z. 2 (1918), 187-197.

[15] R. Westwick, A theorem on numerical range, Linear Multilin. Algebra 2 (1975), 311-315. 